\title{
Recognition using Multimodal Biometric
}

\author{
Shivani Bhardwaj \\ Student, M.Tech (CE) Department \\ UCOE, Punjabi University \\ Patiala, Punjab, India
}

\author{
Supreet Kaur Gill \\ Assistant Professor, \\ M.Tech (CE) Department \\ UCOE, Punjabi University \\ Patiala, Punjab, India
}

\begin{abstract}
In recent time, biometric have been utilized broadly for verification and authentication purposes. Numerous changes and advances have been executed are as yet being created. Some issues in unimodal like absence of comprehensiveness, interclass variety and affectability to assaults which prompt spoofing of the verification framework are resolved by multimodal biometric system. Multimodal biometric system combines more than one biometric trait to authenticate a person's identity. In this paper three biometric traits face, Iris and fingerprint have been combined to develop a secure biometric system. Matching level fusion has been done on traits. Proposed system is more secure since it is using FastIca over face, Hough transformation on Iris. Face acknowledgment is most natural and satisfactory in personality confirmation; Iris and fingerprint have unique features and more accurate. Also Fuzzy logic is applied on the fusion of these three biometric traits.
\end{abstract}

\section{Keywords}

Multimodal biometrics, Fast ICA, Hough transformation, Fuzzy logic

\section{INTRODUCTION}

Biometric authentication, which identifies an individual person using a single biometric trait for authentication using physiological or behavioural characteristics, is one of the most reliable and capable than knowledge-based or token-based techniques, since biometric features are hardly stolen or forgotten[1].However, recognition based on any one of these modalities may not be sufficiently robust or else may not be acceptable to a particular user group or in a particular situation or instance. Therefore, the performance of single biometric system need be improved, and the techniques of multimodal biometric system can offer a feasible method to solve the problems coming from single biometric system [2,3].

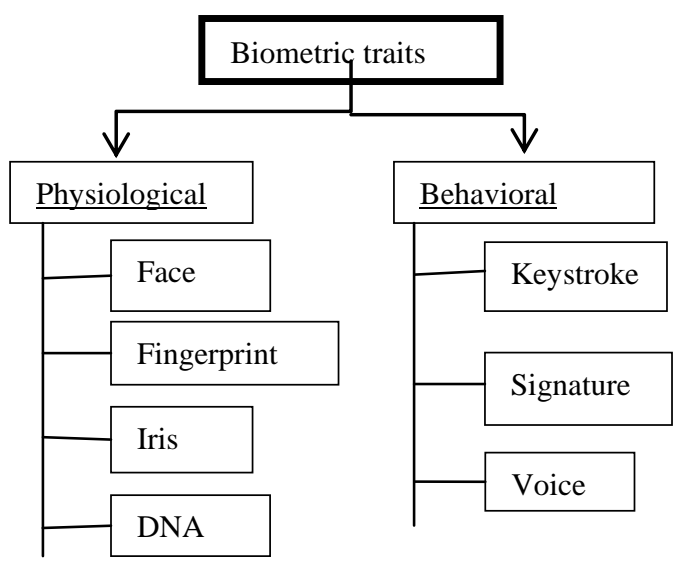

Fig: 1: types of biometrics
Table 1: Comparison of biometric traits

\begin{tabular}{|c|c|c|}
\hline $\begin{array}{l}\text { Biometric } \\
\text { Traits }\end{array}$ & strength & Weakness \\
\hline $\begin{array}{l}\text { Fingerprint } \\
\text { recognition }\end{array}$ & $\begin{array}{ll}\text { - } & \text { Very high } \\
\text { - } & \text { accuracy } \\
\text { Small storage } & \text { space. } \\
\text { - } & \text { Easy to use } \\
\text { - } & \text { Unique } \\
& \text { fingerprints } \\
\text { that do not } \\
\text { change } \\
\text { naturally } \\
\text { It is } \\
\text { standardized }\end{array}$ & $\begin{array}{l}\text { - For some } \\
\text { people it is } \\
\text { very } \\
\text { intrusive, } \\
\text { because it is } \\
\text { still related } \\
\text { to criminal } \\
\text { identification } \\
\text { - } \\
\text { Some people } \\
\text { have } \\
\text { eliminated or } \\
\text { damaged } \\
\text { fingerprint. }\end{array}$ \\
\hline $\begin{array}{l}\text { Facial } \\
\text { recognition }\end{array}$ & $\begin{array}{ll} & \text { Perfectly } \\
\text { designed } \\
\text { system } \\
\text { installed in } \\
\text { banking, } \\
\text { airports and } \\
\text { other public } \\
\text { places. } \\
\text { Does not need } \\
\text { any user } \\
\text { interference } \\
\text { Non- intrusive } \\
\text { Cheap } \\
\text { technology }\end{array}$ & $\begin{array}{l}\text { - } 2 \mathrm{D} \\
\text { recognition } \\
\text { is affected by } \\
\text { changes in } \\
\text { lighting, the } \\
\text { person's hair, } \\
\text { the age, and } \\
\text { if the person } \\
\text { wear glasses. } \\
\text { Requires } \\
\text { camera } \\
\text { equipment } \\
\text { for user } \\
\text { identification }\end{array}$ \\
\hline $\begin{array}{l}\text { Iris } \\
\text { scanning }\end{array}$ & $\begin{array}{ll}- & \text { Most accurate } \\
\text { security system } \\
\text { - } \\
\text { Unique and } \\
\text { easy to find } \\
\text { Verification } \\
\text { time is } \\
\text { generally less } \\
\text { than } 5 \text { sec. } \\
\text { Works } \\
\text { normally even } \\
\text { if person wear } \\
\text { glasses and } \\
\text { contact lenses } \\
\text { at the time of } \\
\text { verification }\end{array}$ & $\begin{array}{ll}- & \text { Intrusive } \\
- & \text { Expensive } \\
- & \text { Large } \\
\text { memory is } \\
\text { required }\end{array}$ \\
\hline $\begin{array}{l}\text { Hand } \\
\text { scanning }\end{array}$ & $\begin{array}{ll}- & \text { Totally } \\
\text { resistant to } \\
\text { attempt to fool } \\
\text { the system } \\
\text { Though it } \\
\text { requires special }\end{array}$ & \\
\hline
\end{tabular}




\begin{tabular}{|l|l|l|}
\hline & hardware to \\
use, it can be \\
easily \\
integrated into \\
other devices \\
or systems. \\
It is authorized \\
access
\end{tabular}

Multi-modal biometric system is a refined arrangement of unimodal framework joining the useful measures for the disadvantages confronted in unimodal biometric framework. This system makes use of different biometric traits simultaneously to authenticate a person's identity. There are three main strategies to build multimodal biometric systems [4].The first method is to apply decision fusion which means combining accept or reject decisions of unimodal systems. The second method to construct a multimodal system is using the feature fusion. This means that feature extracted using multiple sensors are concatenated and the last method is using matching fusion. By the utilization of different quantities of biometric modalities may come about very exact and secure biometric recognizable proof framework $[5,6]$.

\section{RELATED WORK}

Ho et al. (2004) recommended a method important to the recognizable proof issue in which a substantial number of classes (personalities) are available. The combination in this methodology happens at the matching stage and the classifiers report a closeness score for every class [7]. Cappelli et al. (2000) recommended fingerprint grouping framework that consolidates a basic classifier by incorporating the scores produced by the two classifiers [8]. Jain et al (2003) used K closest neighbor classifier and an arrangement of 10 neural system classifiers to characterize fingerprints which are displayed to lessen given arrangement of classes [9]. Sarabjit Singh and Amritpal Kaur (2015) evaluate facial representation predicated on factual nearby elements, Local Binary Patterns, for outward appearance acknowledgment [10].O'Connor and Kaushik Roy (2013) uses modified local binary pattern which consolidates both the magnitudes and sign highlights for the change of facial composition characterization execution, is connected [11].Nupriya Goyal (2015) uses two biometric traits using ICA and genetic algo and provides dynamic scheme of combination [12]. Naresh Babu N T and Vaidehi V (2011) use fuzzy logic on Iris to overcome issues of non-uniform enlightenment, rotational irregularities and noise [13]. Mehdi Ghayoumi (2015) applied Fuzzy logic on multimodal system to discover better limit for adaptation [14].

\section{METHODOLOGY}

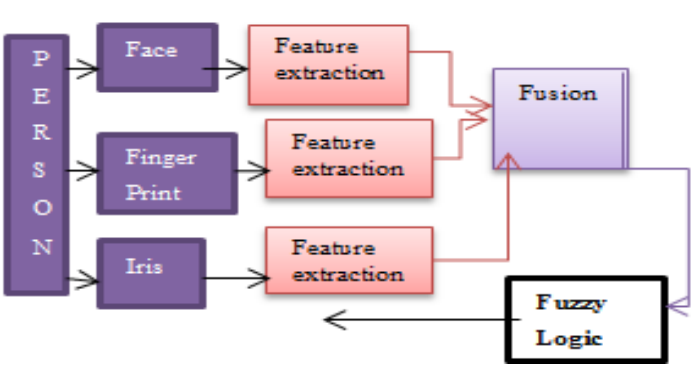

Fig 2: Proposed model

\subsection{Face Recognition}

A facial acknowledgment framework is a PC application for naturally distinguishing or checking a person from an advanced picture or a video outline from a video source. It is a dynamic

examination range, and they can be used in wide range applications. Two types of algorithms are implemented for face recognition.

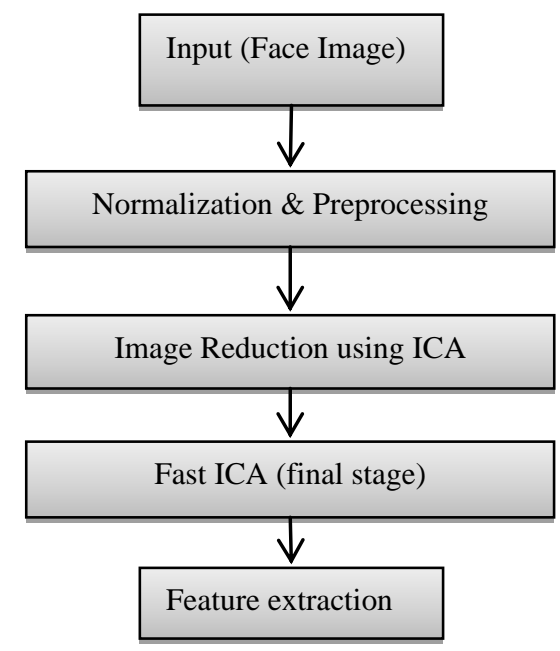

Fig: 3: Flow chart of face recognition using FastICA

\section{a. FastICA}

Independent Component Analysis (ICA) is a measurable and computational method for revealing shrouded components that underlie sets of arbitrary variables, estimations, or signs [15].

FastICA is maybe the most proficient ICA calculation as in it is quick and there are no movable parameters. This calculation depends on the exceptionally essential supposition of ICA that the blended free variables cannot have Gaussian circulation. FastICA has a tendency to boost the measure of nonGaussianity for the information dataset in this way yielding autonomous segments.

\section{Steps [16]:}

1. A filtered image is randomly selected.

2. Find the associated matrix A and Independent component $s$ with given measurement value $\mathrm{X}$ as:

$$
\mathrm{X}=\mathrm{As}
$$

3. Computing the pre-processing steps for ICA

a) Centring the image by removing the mean from $X$

b) Whitening $\mathrm{X}$ by un correlating the components.

4. Computing fixed point iteration for one unit by choosing random vector $\mathrm{w}$ and estimation of $\mathrm{w}$ is repeated until a convergence is achieved.

$\mathrm{s}=\mathrm{w}^{\mathrm{t}} \mathrm{X}$
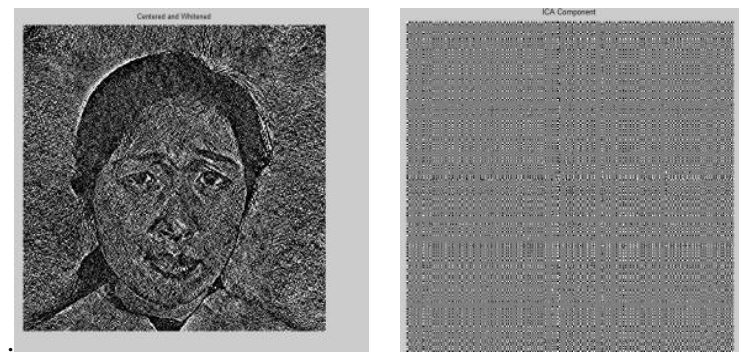

Fig 4: (a) Centering and whitening (b) ICA component 


\section{b. CSLBP}

The linear binary pattern (LBP) was initially intended for texture depiction. It's invariant to monotonic dark scale changes which are crucial for surface depiction also; investigation for the reason of computational effortlessness handling of picture progressively is conceivable.

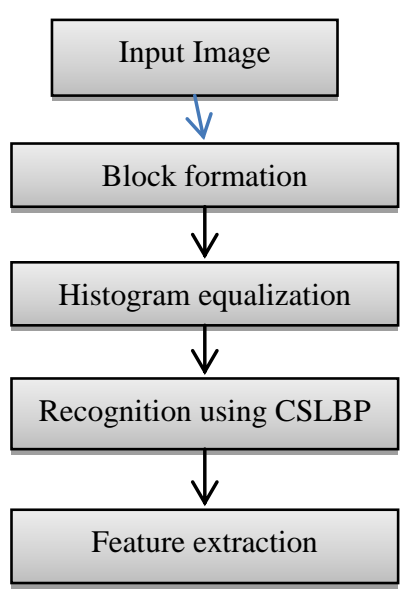

Fig 5: Flowchart of face recognition using CSLBP

\section{Steps:}

i. The facial picture is partitioned into neighborhood areas and LBP surface descriptors are removed from every area freely.

ii. The descriptors are then linked to shape a worldwide portrayal of the face.

iii. Compare centre-symmetric sets of pixels.

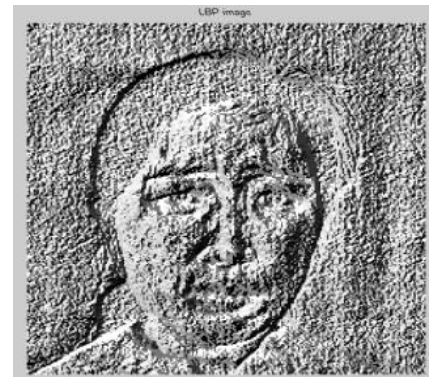

Fig 6: CSLBP image

\subsection{Fingerprint}

Among all the biometric strategies, unique finger impression based distinguishing proof is the most seasoned strategy which has been effectively utilized as a part of various applications. Everybody is known not interesting, changeless fingerprints [17]. A unique mark is made of a progression of edges and wrinkles on the surface of the finger. The uniqueness of a unique mark can be dictated by the example of edges and wrinkles and the particulars focuses. Details focuses are neighbourhood edge qualities that happen at either an edge bifurcation or an edge finishing.

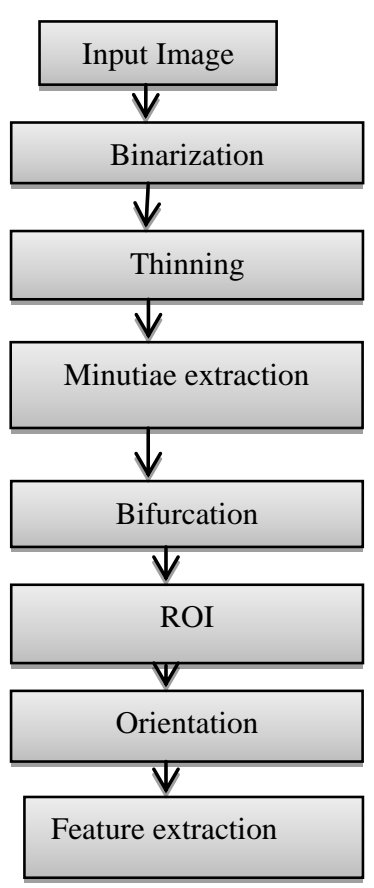

Fig 7: Flowchart of fingerprint recognition

Steps:

1. Image is scanned and enhancement is done if required. In our case, the quality of the image is really good, and we won't need to enhance our image.

\section{Binarize the image}

After the operation, edges in the unique mark are highlighted with dark shading while wrinkle are white.

\section{After that thinning of image is done}

Edge thinning is to dispense with the repetitive pixels of edges till the edges are only one pixel wide.

\section{Minutiae features are then extracted from image}

We filter the diminished edge map by the filter "minutiae". "minutiae" register the quantity of one-estimation of each $3 \times 3$ window: if the focal is 1 and has just 1 one-esteem neighbor, then the focal pixel is a termination, if the focal is 1 and has 3 one-esteem neighbor, then the focal pixel is a bifurcation and if the focal is 1 and has 2 one-esteem neighbor, then the focal pixel is a typical pixel.

5. Bifurcation of image is processed.

\section{Region Of Interest is done}

For determining ROI we consider the binary image and apply a closing on this image and erosion. With the GUI, I allow the use of ROI tools of MATLAB, to define manually the ROI.

\section{Once we get ROI, Orientation is done on image}

There are two ways for implementing orientation: termination and bifurcation.

Termination Orientation: for this investigate the position of the pixel on the limit of a $5 \times 5$ bounding box of the end and contrast this position with the Table variable. The Table variable gives the point in radian.

Bifurcation Orientation: For every bifurcation, we have three lines. So we work the same process than in end case three times. 
i. Once orientation process has been done the features can be extracted

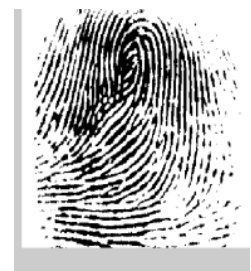

Fig 8: (a) Binarization (b) thinning

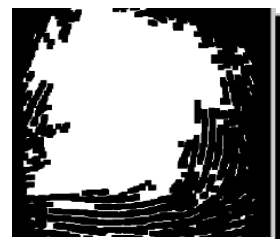

(d) ROI extraction

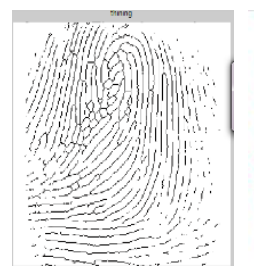

\section{(c)}

(c) minutiae

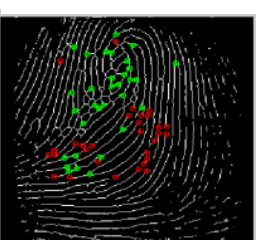

(e) bifurcation orientation

\subsection{IRIS}

Iris is an inside organ of the eye, which is very much ensured from nature and its patterns are obviously steady for the duration of the life. Iris Recognition security frameworks are considered as a standout amongst the most precise security frameworks. It is one of a kind and simple to recognize a client [18]. There must be no physical contact between the client and the framework amid the confirmation process. At the time of verification, if the clients are wearing embellishments, for example, glasses and contact lenses, the framework will work as ordinary since it doesn't change any attributes of the client's iris. Hypothetically, regardless of the fact that clients have eye surgery, it will have no impact on the iris attributes of that person.

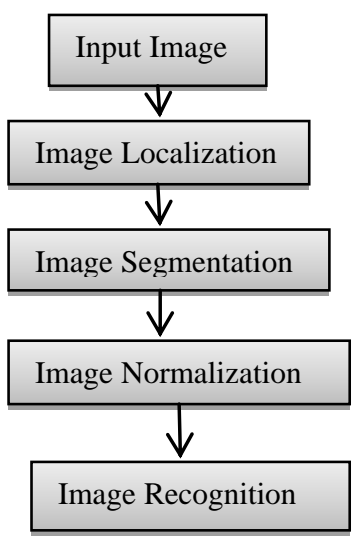

Fig 9: flowchart of Iris Recognition

\section{Steps:}

\section{Image Localization}

Iris image must be preprocessed to distinguish the iris, which is an annular segment between the pupil and the sclera. The initial phase in iris localization is to identify pupil which is the dark round part encompassed by iris tissues. Hough transform has been implemented for pupil detection. Hough Trans form is a standard PC calculation that can be utilized to decide the parameters of straightforward geometric items, Such as lines and circles in a picture.

\section{Image segmentation}

Image segmentation is the procedure of decomposing the picture into the regions also, protests by labeling or associating every pixel with the object that it compares to. Henceforth, segmentation sub partitions a picture into its constituent locales or items. It is done by morphology.

\section{Image Normalization}

To cop up with the radial size of pupil, normalization of image is done. In this segmented image is transformed from cartesian to polar coordinates[19].

\section{Recognition}

Done using Euclidian Distance.
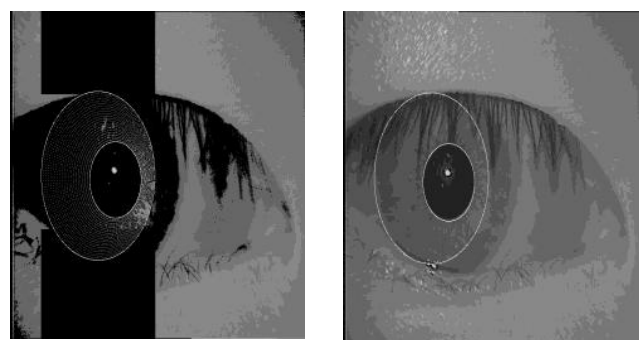

Fig 10: (a) segmentation (b) localization

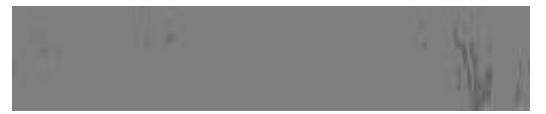

(c) normalization

\subsection{Fuzzy Logic}

Fuzzy logic is a form of many-valued logic in which the truth values of variables may be any real number between 0 and 1 , considered to be "fuzzy". By contrast, in Boolean logic, the truth values of variables may only be 0 or 1 , often called "crisp" values. Fuzzy logic has been extended to handle the concept of partial truth, where the truth value may range between completely true and completely false. Fuzzy logic is reasonably straightforward, adaptable and can mix with traditional system. Fuzzy logic has been connected to numerous fields, from control hypothesis to computerized reasoning. Weightings can be alternatively added to every tenet in the rulebase and weightings can be utilized to manage the extent to which a standard influences the yield values. These principle weightings can be based upon the need, dependability or consistency of every guideline. These tenet weightings might be static or can be changed powerfully [13].

\subsubsection{Fuzzy Process}

1. Fuzzify all info values into fuzzy membership functions.

The membership function is a graphical representation of the greatness of cooperation of every info. It relates a weighting with each of the inputs that are handled, characterize useful cover amongst inputs, and at last decides a yield reaction.There are distinctive membership function connected with every data and yield reaction.

2. Execute all relevant principles in the rulebase to figure the fuzzy yield functions.

The essential goal of this develop is to outline the universe of conceivable inputs while holding the framework adequately under control.

3. De-fuzzify the fuzzy yield capacities to get "fresh" yield values.

The defuzzification of the information into a fresh yield is expert by joining the aftereffects of the deduction procedure 
and after that processing the "fuzzy centroid" of the region. The weighted qualities of every yield part capacity are duplicated by their individual yield enrollment capacity focus focuses and summed. At long last, this zone is separated by the aggregate of the weighted part work qualities and the outcome is taken as the fresh yield [20].

Table 2: Fuzzy rule

\begin{tabular}{|l|l|l|l|}
\hline Face & IRIS & Fingerprint & Authentication \\
\hline 0 & 0 & 0 & No \\
\hline 0 & 0 & 1 & N0 \\
\hline 0 & 1 & 0 & no \\
\hline 0 & 1 & 1 & yes \\
\hline 1 & 0 & 0 & no \\
\hline 1 & 0 & 1 & yes \\
\hline 1 & 1 & 0 & yes \\
\hline 1 & 1 & 1 & yes \\
\hline
\end{tabular}

\section{EXPERIMENTAL RESULTS}

In this area, the execution of work is examined. The investigations are performed on TPR and TNR parameters individually on face, fingerprint and iris. Jeffe database for face, CASIA database for fingerprint and Iris utilized here. Acknowledgment of face and fingerprint has been done utilizing chi-square exclusively and Euclidian Distance for Iris. Testing and training has been performed on three traits. Results show that true positive rate of face, fingerprint and Iris comes to be $88 \%, 86 \%$ and $90 \%$ respectively shown in table 3.After that matching level fusion done on these three traits and on applying fuzzy logic recognition rate comes to be $92 \%$ making this system more accurate shown in figure 11 as graph. Chi square is any statistical theory test wherein the examining appropriation of the test measurement is chi-square dispersion when the null speculation is valid. Chi-squared tests are frequently developed from a sum of squared errors.

Table 3: showing performance

\begin{tabular}{|l|l|l|l|}
\hline Traits & Images & TPR & TNR \\
\hline Face & 50 & 0.88 & 0.12 \\
\hline Fingerprint & 25 & 0.86 & 0.14 \\
\hline Iris & 25 & 0.90 & 0.10 \\
\hline $\begin{array}{l}\text { Combined(Face+Iris+ } \\
\text { Fingerprint) }\end{array}$ & 25 & 0.92 & 0.8 \\
\hline
\end{tabular}
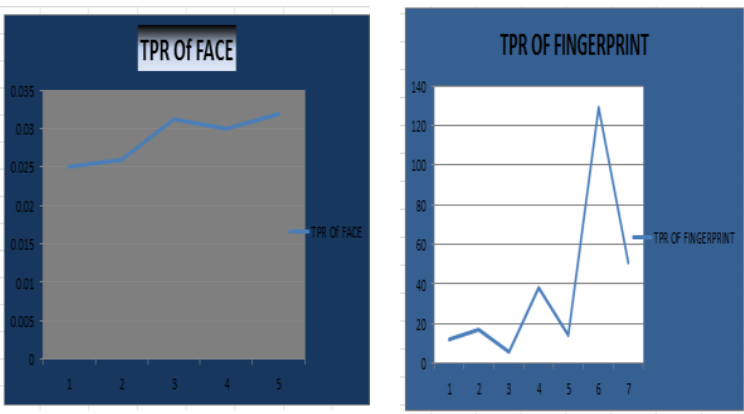

Fig 11: graph showing TPR of (a) face (b) fingerprint

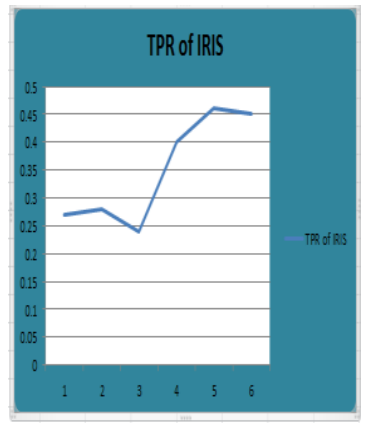

(c) Iris

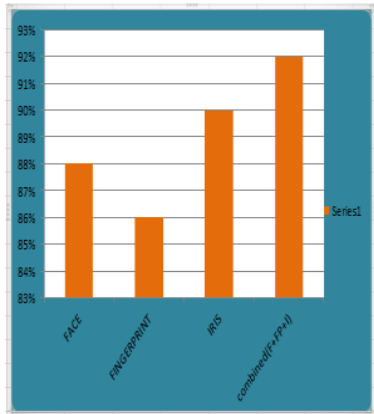

(d) overall result of system

\section{CONCLUSION AND FUTURE SCOPE}

Biometric frameworks offer a few focal points over conventional based techniques. This work concentrates on utilizing the multimodal biometrics by using face, fingerprint and Iris. The individual scores of three attributes are consolidated at coordinating level to build up a multimodal system. Final acknowledgment is done utilizing type 1 fuzzy logic and shows great exactness. In future work, the execution can be expanded by utilizing different sorts of FastICA, additionally by utilizing other kind of Fuzzy logic.

\section{REFERENCES}

[1] A. K. Jain, A. Ross, and S. Prabhakar, "An Introduction to Biometric Recognition", IEEE Trans. on Circuits and Systems for Video Technology, vol. 14, no. 1, pp. 4-20, 30, Jan. 2004.

[2] Nazia Nafis, "A review on the Existent and Emerging Biometrics Modalities", AEICT-2014.

[3] Olufemi Sunday Adeoye, "A survey of Emerging Biometric Technologies", International Journal of Computer Appliccations (0975- 8887), Vol. 9, No. 10, November 2010

[4] Anil k.Jain and Arun Ross "Multimodal biometric systems", Communication of THE ACM January 2004/Vol. 47, No. 1

[5] Ashish Mishra "Multimodal biometrics it is: need for future systems" International journal of computer applications volume 3-No.4, June 2010.

[6] A. K Jain, A. Ross, "Information Fusion In Biometrics",Elsevier, Pattern Recognition Letters 24 (2003)

[7] Ho T.K, Hull J.J, Srihari S.N et al.Decision combination in multiple classifier systems [J]. IEEE Trans. PAMI 1994, 16 (1): 66- 75 
[8] Cappelli, R., Maio. D, Maltoni D et al.Combining fingerprint classifiers. In First Internet. Workshop on Multiple Classifier Systems, 2000: 351-361

[9] A. Ross, A.K.Jain, et al. Information fusion in biometrics. Pattern Recognition Letters, 2003, 24(13): 2115 -2125.

[10] Sarabjit Singh, Amritpal Kaur "A Face Recognition Technique using Local Binary Pattern Method" IJARCCE Vol. 4, Issue 3, March 2015

[11] Brian O'Connor and Kaushik Roy "Facial Recognition using Modified Local Binary Pattern and Random Forest" (IJAIA), Vol. 4, No. 6, November 2013

[12] Nupriya Goyal “ Multi-Biometric Recognition for Iris and Ear Images by using ICA, GA, Hamming Distance" JNCET Volume 2, Issue 1, May (2015)

[13] Naresh Babu N T, Vaidehi V "Fuzzy Based IRIS Recognition System (FIRS) For Person Identification" IEEE- ICRTIT 2011.

[14] Mehdi Ghayoumi "An Adaptive Fuzzy Multimodal Biometric System for Identification and Verification IEEE-ICIS 2015, June 28-July 1 2015, Las Vegas, USA.
[15] Tanmay Awasthy, Anubhav Kumar "Analysis of FastICA Algorithm for Separation of Mixed Images", IJECSE, ISSN 2277-1956

[16] Li-Yuan Chen and Chi-Jie Lu "An Improved Independent Component Analysis Algorithm Based on Artificial Immune System", DOI: 10.7763, IJMLC.2013.V3.279

[17] Samarth Bharadwaj, Mayank Vatsa "Biometric quality: a review of fingerprint, iris and face", EURASIP Journal on Image and Video Processing 2014

[18] P. Richard, Wildes, "Iris Recognition: An Emerging BiometricTechnology", Proc. of the IEEE Int'l conf. on image processing, vol. 85, no. 9, September 1997.

[19] L. Flom and A. Safir, "Iris Recognition System", US Patent 4641394, 1987

[20] Chung-Chih Tsai, Heng-Yi Lin "Iris Recognition Using Possibilistic Fuzzy Matching on Local Features" IEEE transactions on systems, man, and cybernetics-part b: cybernetics, vol. 42, no. 1, February 2012 